\title{
Application of Inverse Fuzzy Logical Inference to Breakdown Diagnosis
}

\author{
Jeng-Jong Lin \\ Department of Information Management, Vanung University, Chung-Li, Tao-Yuan, Taiwan, R.O.C.
}

\begin{abstract}
An intelligent diagnosis methodology, which can trace the possible breakdown causes, is developed using fuzzy set theory in this paper. Application of the inverse logical inference in the expert systems of diagnosis is considered. Diagnosis decision finding requires fuzzy logical equations system solution. The diagnosis based on fuzzy logical equations can act as an expert consultant to facilitate the operator to trace the causes of breakdown at any time.
\end{abstract}

Keywords: Intelligent diagnosis, Fuzzy set, Breakdown causes, Inverse logical inference

\section{Introduction}

At present, the inspection processing and the tracing breakdowns that occur during process of manufacturing still heavily depend on the experience of the technical operator. It is not only time-consuming but also economically infeasible for an enterprise to retrain a new operator to expert at the specific technical knowledge of spinning engineering once the trained operator leaves the job.

Fuzzy sets theory is a handy tool for expert information formalization while simulating cause-effect connections in technical and medical diagnostic problems [1]. The model of a diagnostic object, as a rule, is built on the basis of compositional Zadeh rule of inference which connects input and output variables of an object (cause and effects) using fuzzy relational matrix [2]. The problem of diagnosis can be formulated in the form of the direct and inverse fuzzy logical inference. The direct logical inference suggests finding diagnoses (output variables or effects) according to observable internal parameters of the object state (input variables or causes). In the case of the inverse logical inference some renewal of causes takes place (of the object state parameters) according to observable effects (symptoms). In the majority of fuzzy logic applications to the diagnosis problems the direct logical inference is used [1,2]. At the same time the inverse logical inference is used much less duo to the lack of effective algorithms solving fuzzy logical equation system .

In this paper, we present a search model, which divide symptoms into two sets, i.e., the positive symptom set $\left(\mathrm{J}_{1}\right)$ and the negative symptom set $\left(\mathrm{J}_{2}\right)$, to eliminate the causes of low possibility in the cause set to more effectively find various possible breakdown causes occurred during spinning process.

\section{Fuzzy Logic Equation}

The diagnosis object is treated as the black box with $\mathrm{n}$ inputs and $\mathrm{m}$ outputs:

$$
\begin{aligned}
& X=\left(x_{1}, x_{2}, \ldots, x_{m}\right)-\text { set of inputs; } \\
& Y=\left(y_{1}, y_{2}, \ldots, y_{n}\right)-\text { set of outputs. }
\end{aligned}
$$

Simulation of the cause-effect "input-output" connections is done by way of interpreting the compositional Zadeh's rule of inference [3]

$$
\mathrm{A} 。 \mathrm{R}=\mathrm{B}
$$

where

$$
\begin{aligned}
& R=\left(\begin{array}{cccc}
r_{11} & r_{12} & \ldots & r_{1 n} \\
r_{21} & r_{22} & \ldots & r_{2 n} \\
\cdot & \cdot & \\
r_{m 1} & r_{m 2} & r_{m n}
\end{array}\right) \\
& A=\left(a_{1} a_{2} \ldots a_{m}\right) ; B=\left(b_{1} b_{2} \ldots b_{n}\right)
\end{aligned}
$$

Where A and B are fuzzy sets given on the universal sets $X$ and $Y ; a_{i}$ and $b_{j}$ are degrees of membership, that is of the numbers in the diapason from o to 1 interpreted as some measures of $x_{i}$ causes and $y_{j}$ effects significances; $\mathrm{R}$ is the fuzzy relational matrix with elements $r_{i j}$, which is the number in the range of $[0,1]$ characterizing the degree of the cause $\mathrm{xi}$ influence on the rise of the effect $\mathrm{y}_{\mathrm{j}}$; 。 is the operation of max-min composition [4].

The diagnostic problem is set in the following way. According to the known matrix R and fuzzy set $\mathrm{B}$ it is necessary to find some fuzzy set A. It is suggested that matrix R and fuzzy set B be formed on the basis of expert assessments, for example, by way of paired comparisons:

The relationship between causes and symptoms in a diagnosis problem can thus be shown as follows.

$$
\underset{\mathrm{i}}{\mathrm{V}}\left(\mathrm{a}_{\mathrm{i}} \Lambda \mathrm{r}_{\mathrm{ij}}\right)=\mathrm{b}_{\mathrm{j}}
$$

where $\mathrm{V}: \max , \quad \Lambda$ :min, $\mathrm{i}=1,2, \ldots, \mathrm{m}, \quad$ and $\mathrm{j}=1,2, \ldots, \mathrm{n}$.

The diagnostic procedure seems quite simple using given matrix $\mathrm{A}$ and matrix $\mathrm{R}$ to find the solution of matrix $\mathrm{B}$, because there exists only one specific solution. Yet using matrix $B$ and $R$ to find matrix $\mathrm{A}$, which can fit the requirement of equation 1 , will be rather more sophisticated because more than one solution exists. The diagnostic procedure, usually 
proceeding with given matrices $\mathrm{R}$ and $\mathrm{B}$ to find the solution of matrix $\mathrm{A}$ that fits the requirements of Equation 1, is an inverse problem of fuzzy relation equation. If the solution of matrix A can be found, then the breakdown cause is obtained.

\section{Solutions to the Inverse Problems}

Assuming that matrices A, B, and R in Equation 1 are all fuzzy set, to find the solution of matrix $\mathrm{A}$ in Equation 1 from given matrices $\mathrm{B}$ and $\mathrm{R}$ is an inverse problem of a fuzzy relational equation. For instance, when $m=n=1$, the solution, represented as $a^{*}$, of the inverse problem of $b=a \Lambda r$ can be shown as

$$
\left\{\begin{array}{lll}
\mathrm{a}^{*}=\mathrm{b} & \text { if } & \mathrm{b}<\mathrm{r} \\
\mathrm{a}^{*}=[\mathrm{b}, 1] & \text { if } & \mathrm{b}=\mathrm{r} \\
\mathrm{a}^{*}=\phi & \text { if } & \mathrm{b}>\mathrm{r}
\end{array}\right.
$$

Relationships between $\mathrm{b}$, r, and a can be illustrated as in Figure 1, from which we can conclude that when $b<r$ and $b=r$, it is true for $a=b$ and $a=[b, 1]=[r, 1]$ respectively. But when $b>r$ because there is no a, the solution is $\phi$. In accordance with the magnitudes of $b$ and $r$, there exist three kinds of solutions (i.e., point, set, and $\phi$ ). From Figure 1, we can conclude that a solution exists for $b=a \Lambda r$ unless the magnitude of $r$ is less than that of $b$.

The effective algorithm for solving the inverse problem have been researched and reported in many studies [4]. We first obtain a concise method for determining all the minimal solutions [2] using Pappis and Sugeno's method [4] to find all the solution sets of Equation 1. Then from each of the solution sets, we acquire a minimal solution. The minimal solution may be the only one obtained, but it may not be the only one available. If not, finding a virtual minimum solution, which can be acquired by allowing all minimal solutions to be processed by min operator, is deemed necessary. The major steps of the algorithm illustrate as follows.

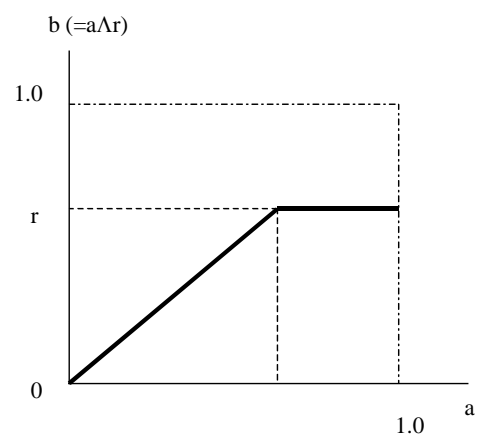

$\underline{\text { Step } 1}$ Figure 1 Graph of $b=a \wedge r$

Compare all the elements of each column in matrix $\mathrm{R}$ to the element of the same column in matrix $B$. If the value of the element in matrix $R$ is not larger than that of the element in matrix $B$ (namely $r_{i j}$ $\leq b_{j}$ ), then we change the value of the element in matrix $\mathrm{R}$ into 1 , or else into that of the responding element in matrix $B$ to obtain matrix $R_{1}$. Then we allow all the elements of each row in matrix $R_{1}$ to be processed by min operator to obtain a column matrix $\mathrm{U}$, which is the least upper boundary (L.U.B.) [4] of the solution vectors of the inverse problem.

\section{Step 2}

Compare all the elements of each column in matrix $\mathrm{R}$ to the element of the same column in matrix $B$. If the value of the element in matrix $R$ is greater than or equal to that of the element in matrix $\mathrm{B}$ (namely $r_{i j} \geq b_{j}$ ), then change the value of the element in matrix $R$ to $b_{j}$, or else to 0 to obtain a matrix $R_{2}$.

$\underline{\text { Step } 3}$

Compare all the elements of each row in matrix $R_{2}$ to the element of the same row in matrix $U$. If the value of the element in matrix $R_{2}$ is greater than that of the element in matrix $U$ (namely $r_{2 i j}>u_{i}$ ), then change the value of the element in matrix $R_{2}$ to 0 , or else leave it as it was originally to obtain a matrix W.

\section{$\underline{\text { Step } 4}$}

Check to see if the elements of each column in matrix $\mathrm{W}$ are 0 all the same. If there exists any column like that in matrix $\mathrm{W}$, then conclude that there is no solution existing in the fuzzy relation equation. But if not, then conclude the existence of a solution.

\section{Step 5}

If the existence of a solution is concluded, then take an element that is not 0 from each column in matrix $\mathrm{W}$ to form a new matrix. Each matrix obtained is a minimal solution of the inverse problem.

From given matrices $\mathrm{R}$ and $\mathrm{B}$, as shown below, the results for calculating the inverse problem of a fuzzy relation equation using the algorithm of step 1-5 are illustrated in Figure 2.

\section{Effective Search Model}

In order to develop an intelligent diagnosis system, which is capable of tracing the possible breakdown causes from the categories of defects and providing an immediate response, it is necessary to sketch an effective searching algorithm for the diagnosis procedure. Firstly, we define the following symbols:

$A_{i}=\left\{a_{1}, a_{2}, \ldots . ., a_{m}\right\}=$ cause set

$\mathrm{B}_{\mathrm{j}}=\left\{\mathrm{b}_{1}, \mathrm{~b}_{2}, \ldots \ldots, \mathrm{b}_{\mathrm{n}}\right)=$ symptom set

$\mathrm{R}_{\mathrm{ij}}=\left(\mathrm{r}_{\mathrm{ij}}\right)_{\mathrm{mxn}}=$ fuzzy relation matrix of size $\mathrm{m} \times \mathrm{n}$ between $\mathrm{x}$ and

where

$a_{1}-a_{m}: m$ kinds of breakdown causes, 
$b_{1}-b_{n}: n$ kinds of symptoms, and

$r_{i j}$ : the fuzzy truth value between the ith kind of cause and the jth kind of symptom.

The fuzzy truth values of $r_{i j} s$ are acquired empirically from experts of engineering using the following linguistic values (e.g., completely true, very true, true, rather true, rather rather true, and unknown) of the linguistic variable "truth." Their meaning is defined as follows.

$$
\begin{aligned}
& R=\left(\begin{array}{ccc}
0.2 & 1 & 0 \\
0.6 & 0.4 & 0.2 \\
0.1 & 0 & 1
\end{array}\right) \quad B=\left(\begin{array}{ll}
0.6 & 0.8
\end{array}\right. \\
& \mathrm{R}_{1}=\left(\begin{array}{ccc}
1 & 0.8 & 1 \\
1 & 1 & 1 \\
1 & 1 & 0.8
\end{array}\right) \\
& \mathrm{U}=\left(\begin{array}{c}
0.8 \\
1 \\
0.8
\end{array}\right)
\end{aligned}
$$

(a)

$$
\mathrm{R}_{2}=\left(\begin{array}{ccc}
0 & 0.8 & 0 \\
0.6 & 0 & 0 \\
0.6 & 0 & 0.8
\end{array}\right) \quad \mathrm{W}=\left(\begin{array}{ccc}
0 & 0.8 & 0 \\
0.6 & 0 & 0 \\
0.6 & 0 & 0.8
\end{array}\right)
$$

(c)

Minimal solutions:

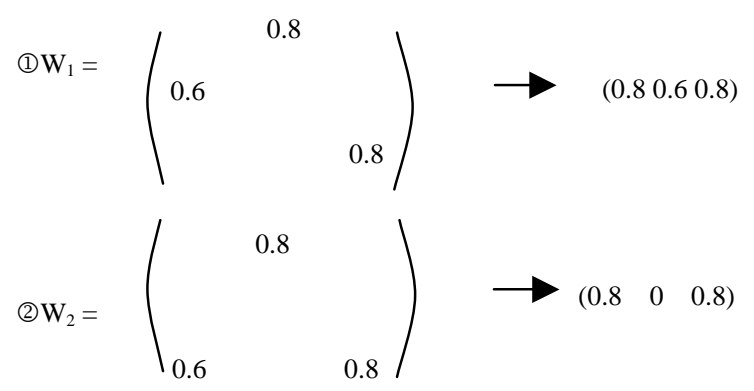

(e)

Virtual minimum solution : (0.8 0.8 0.6)

Figure 2 Calculations from steps 1-5

(1) completely true : Once $a_{i}$ occurs then $b_{j}$ appears.

(2) very true : When $a_{i}$ occurs, $b_{j}$ will appear very definitely.

(3) true: When ai occurs, bj will appear very probably.

(4) rather true: When ai occurs, bj will appear probably.

(5) rather rather true : When $a_{i}$ occurs, $b_{j}$ will appear seldom.

(6) unknown : When $a_{i}$ occurs, $b_{j}$ will never appear.

Generally speaking, in a diagnosis problem, the symptoms can be divided into two kinds of categories, the positive symptom set $\left(\mathrm{J}_{1}\right)$, consisting of those symptoms that have been observed by the operator, and the negative symptom set $\left(\mathrm{J}_{2}\right)$, consisting of those symptoms that have not yet been observed by the operator. When only certain symptoms have been observed by the operator, the diagnosis process can proceed. It is impossible for all the symptoms of the system to appear at one time, so that $\mathrm{J}_{1} \neq \phi$ and $\mathrm{J}_{2} \neq \phi$.

Actually during tracing a certain kind of breakdown cause through the observed symptoms, the reliability of diagnostic results should be very high as long as all possible symptoms for this kind of breakdown are all observed. However, if there are many other symptoms (not the observed ones) that should have appeared but have not yet done so, then the reliability of diagnostic results of this kind of breakdown cause will be very low.

We can thus conclude that the diagnostic range can be narrowed effectively by neglecting those breakdown causes seldom noticed $\mathrm{a}_{\mathrm{i}}$. For instance, breakdown causes that are in accordance with the circumstance of

$$
\underset{j \in J 2}{V_{i j}} R_{i j} \text { rather rather true, }
$$

should firstly be investigated. That is, the searching range of the diagnosis can be narrowed from $i \in I$ $(=\{\mathrm{i} \mid \mathrm{i}=1,2, \ldots, \mathrm{m}\})$ down to

$$
\mathrm{i} \in \mathrm{I}_{1}\left(=\left\{\mathrm{i} \mid \mathrm{V} \mathrm{R}_{\mathrm{j} \in \mathrm{Jj}}<\text { rather rather true }\right\}\right) .
$$

A relationship should occur between the breakdown causes searched $a_{i}$ and the observed symptoms $b_{j}$. In other words, the condition of

$$
\underset{j \in J 1}{V_{i j}}>\text { unknown }
$$

should be true. Therefore the searching range of diagnosis $I_{1}$ can be reconstructed as $I_{1}=\left\{i \mid V R_{i j}<\right.$ rather rather true, $\underset{j \in J 2}{V_{\mathrm{ij}}} \mathrm{R}_{\mathrm{i}}$ > unknown $\} . \quad \mathrm{j} \in \mathrm{J} 1$

In a practical diagnostic procedure in the real world, the members in $I_{1}$ are much fewer than those in cause set I (consisting of $\mathrm{m}$ members). Thus, we can obtain an efficient searching method.

Nevertheless, in a practical diagnostic procedure, while searching for the members of the set searching range $I_{1}$, the circumstance of $I_{1}=\phi$ can happen. Then a wider searching range should be reset to search once again. Yet the wider the searching range, the less reliable the breakdown cause found through this diagnostic procedure. In order to achieve both effectively narrowing the diagnostic searching range and specific reliability of the diagnostic result, the extension of the searching range in a diagnosis procedure should have a proper limitation. Therefore, there are three kinds of searching range in this study. These sets and their reliability are represented as

$$
\mathrm{I}_{1}\left(=\left\{\mathrm{i} \mid \mathrm{V} \mathrm{R}_{\mathrm{ij}}<\text { rather rather true, } \mathrm{V} \mathrm{R}_{\mathrm{ij}}>\right.\right.
$$

unknown $\}), \quad j \in J 2 \quad j \in J 1$

which has the greatest reliability and from which the diagnostic result that is found can be regarded as the actual "cause"; unknown\}),

$$
I_{2}\left(=\left\{i||_{j \in J 2}^{V} R_{i j}<\text { rather true, } \underset{j \in J 1}{V} R_{i j}>\right.\right.
$$

which is less reliable than $I_{1}$ and from which the diagnostic result that is found can be regarded as "very probable"; and

$$
\mathrm{I}_{3}\left(=\left\{\mathrm{i} \mid \underset{\mathrm{j} \in \mathrm{J} 2}{\mathrm{~V}_{\mathrm{ij}}} \quad<\underset{\mathrm{j} \in \mathrm{J} 1}{\mathrm{~V} \mathrm{R}_{\mathrm{ij}}}>\text { unknown }\right\}\right),
$$


which is the least reliable, and from which the diagnostic result that is found can be regarded as "probable".

Finally after searching for the members of the searching ranges $I_{1}, I_{2}$, and $I_{3}$ using the effective diagnostic procedure mentioned above, there probably exists the circumstance of $I_{1}=I_{2}=I_{3}=\phi$. Then the system will select three $\mathrm{x}_{\mathrm{i}}$ of greater $\mathrm{L}_{\mathrm{i}}$ value as the suspected breakdown causes for further diagnosis:

$$
\mathrm{L}_{\mathrm{i}}=\sum_{\mathrm{j} \in \mathrm{J}_{1}} \mathrm{R}_{\mathrm{ij}}
$$

where

$\mathrm{R}_{\mathrm{ij}}$ : the fuzzy truth value between the ith kind of breakdown cause and the jth kind of symptom $\mathrm{J}_{1}$ : the positive symptom index set

\section{Implementation}

\subsection{Example}

An application of the intelligent diagnosis methodology to tracing the breakdown cause occurred during spinning was reported in this study. There were seven kinds of defects most likely found during spinning and twenty one possible occurrence causes due to these defects chosen in this study.

The cause set $\mathrm{A}$ and the symptom set $\mathrm{B}$ consist of the above-mentioned twenty one causes and seven kinds of defects respectively and the elements of each of the two are illustrated as below.

\section{SYMPTOMS}

$\mathrm{y}_{1}$ r oller licked with fiber due to static electricity

$\mathrm{y}_{2}$ roller licked with fiber due to oil

$\mathrm{y}_{3} \quad$ cylinder licked with fiber

$\mathrm{y}_{4}$ coiler stocked with fiber

$\mathrm{y}_{5}$ licked lap

$\mathrm{y}_{6}$ slub yarn or shrinkage yarn

$\mathrm{y}_{7}$ neps

\section{CAUSES}

$\mathrm{x}_{1}$ fiber with too low moisture regain

$\mathrm{x}_{2}$ fiber with too large electricity resistance

$\mathrm{x}_{3}$ fiber licked with oil

$\mathrm{x}_{4}$ bulky fiber

$\mathrm{x}_{5}$ too big friction durability

$\mathrm{x}_{6}$ aggregated bulk fiber

$\mathrm{x}_{7}$ too big elongation \& elasticity

$\mathrm{x}_{8}$ too big fiber residence

$\mathrm{x}_{9}$ too big fiber shrinkage

$\mathrm{x}_{10}$ inappropriate humidity tuning

$\mathrm{x}_{11}$ bad material and condition of roller

$\mathrm{x}_{12}$ inappropriate scale of knit/ mal-tuning of roller / improper speed of doffer

$\mathrm{x}_{13}$ improper type of coil

$\mathrm{x}_{14}$ improper setting of silver weight and output speed

$\mathrm{x}_{15}$ improper sliver weight

$\mathrm{x}_{16}$ incomplete cleaning for dust cage

$\mathrm{X}_{17}$ improper wrapping for lap

$\mathrm{x}_{18}$ improper setting of drawing

$\mathrm{x}_{19}$ improper roving twist
$\mathrm{X}_{20}$ improper setting in fiber beating

$\mathrm{x}_{21}$ improper sliver processing

\subsection{Fuzzy Relation Matrix}

The fuzzy truth value of each $r_{i j}$ was acquired empirically from experts of weaving engineering and technical references on causes and effects of the fabric defects in weaving.

\subsection{Implementation Results}

The troubleshooting-aided system involves providing the operator of "on call" expert assistance in a wide variety of information regarding the breakdown occurrence causes. Then a crucial criterion is paramount: the immediacy of service availability can be fulfilled, since the operator frequently needs help "now."

Along with the diagnostic results, the $\mathrm{W}$ matrix and the least upper boundary (L.U.B.) derived from the effective diagnostic algorithm mentioned earlier are obtained, and the display of the result by the diagnosis system is shown in Figure 3.

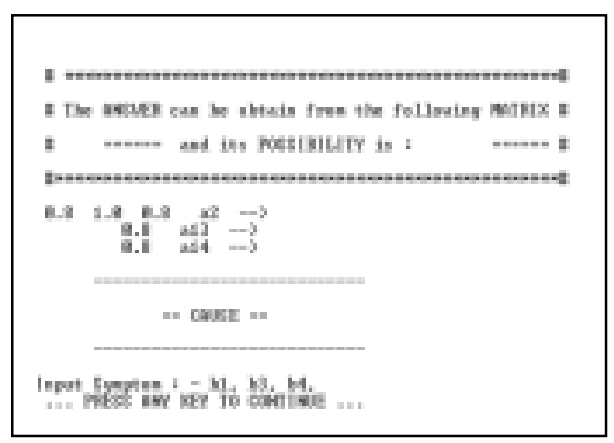

Figure 3 diagnosis results

\section{Conclusions}

Through the assistance of the intelligent diagnosis system, even a new operator, who is lack of the expertise and experience in the spinning engineering field, can still easily find out the occurring causes of the defects occurred during spinning in a fraction of a second and then eliminate them.

\section{References}

[1] Chen, P., Taniguchi, et al. "Intelligent diagnosis method of multi-fault state for plant machinery using wavelet analysis, genetic programming and possibility theory," Proceedings - IEEE International Conference on Robotics and Automation, Vol. 1, 610-615, 2003.

[2] A. Rotshtein, "Design and Tuning of Fuzzy Rule Based Systems for Medical Diagnostics," In: N.-H. Teodorescu A. Kandel (ed.): Fuzzy and Neuro-Fuzzy Systems in Medicine, CRC Press, 243-289, 1998.

[3] Zadeh, L. A., Fuzzy Sets, Inf. Control 8, 338-353, 1965.

[4] Pappis, C. P., and Sugeno, M., Fuzzy Relational Equations and the Inverse Problem, Fuzzy Sets, Vol. 5, 79-90, 1985.

[5] Xu, G. , Luo, Z. , Li, M. , Chen, P., "Mechanical failure diagnosis in unsteady operating conditions", Vol. 37, Issue 12, , 104-107, 2001. 